\title{
Development of a plasma electroacoustic actuator for active noise control applications
}

\author{
S Sergeev ${ }^{1}$, H Lissek ${ }^{1}$, A Howling ${ }^{2}$, I Furno ${ }^{2}$, G Plyushchev ${ }^{2}$ \\ and P Leyland ${ }^{3}$ \\ 1 Signal Processing Laboratory LTS2, EPFL, CH-1015 Lausanne, Switzerland \\ 2 Swiss Plasma Center SPC, EPFL, CH-1015 Lausanne, Switzerland \\ ${ }^{3}$ Group of Thermal Turbomachinery SCI-STI-PO, EPFL, CH-1015 Lausanne, \\ Switzerland
}

E-mail: stanislav.sergeev@epfl.ch, herve.lissek@epfl.ch

\begin{abstract}
Conventional loudspeakers generate sound through the vibration of a diaphragm, attached to a rigid frame through elastic suspensions. Although such construction is satisfactory for sound diffusion in steady environments, it is likely to fail in harsh conditions, which is often the case for active noise control applications. Plasma-based actuators appear to be a promising alternative since they do not involve any fragile moving parts. In this paper, a positive corona discharge actuator in a wireto-mesh geometry is proposed in the perspective of active noise control applications, as it is capable of generating sufficient sound pressure levels with limited signal distortion. The study introduces analytical and numerical models aiming at characterizing the sound field generated by the corona discharge actuator. The numerical simulation can be used for designing such transducers without requiring a calibration with experiment. The acoustic power of the experimental prototype is increased through the optimization of emitter wires arrangement. The comparison of analytical model and numerical simulation with the experiment is presented. The analytical model successively describes the low frequency sound pressure field, while the numerical simulation is valid in the broader frequency range.
\end{abstract}

Keywords: Corona discharge, plasma loudspeaker, electroacoustics, active noise control, nonlinear distortion

Submitted to: J. Phys. D: Appl. Phys.

\section{Introduction}

Environmental noise mostly related to human activities has a huge impact on public health [1]. As a consequence, modern stringent regulations were adopted to control the growing noise pollution. The need for a breakthrough in the noise reduction technologies is now driving many industries, especially the aircraft sector, where the harsh environment and the limited space for acoustic treatment along with the complex noise spectra further complicate the task. 
Beyond the conventional passive noise control materials, (porous medium, resonators, silencers, etc.) which are limited in their performance at low frequencies $[2,3]$, active noise control techniques raise a high research interest, and already find concrete industrial applications $[4,5]$. Various approaches have been reported in the literature so far. Active pressure cancellation can be used to minimize the sound pressure level at an arbitrary point in space [6,7]. Broadband sound absorption can be achieved by direct active acoustic impedance control $[8,9]$ or by combining passive and active methods, where the active element acts as a sink for sound waves, inducing energy loss through a (passive) resistive layer $[10,11]$. Such concepts have been successfully validated in grazing flow-duct facilities for aircraft engine noise reduction $[11,12]$. The majority of these studies utilize membrane-based electroacoustic transducers (mostly electrodynamic loudspeakers) as the controlled actuators to convert an electric command into an acoustic response. The dynamic behaviour of conventional loudspeakers can be well captured by simple lumped-element analytical models [13], which makes these transducers a favorable choice for many control applications. However, this solution can be less suitable in certain situations. For instance, when a large number of transducers is required, (e.g. to cover a large surface [12]), the weight of the total system can become unacceptable. Furthermore, active control solutions are likely to be required in harsh environments with not only high Sound Pressure Levels (SPL), but also extreme climatic conditions. Since a loudspeaker membrane is typically composed of cellulose, it may not sustain such extreme loads. Piezoelectric actuators are an alternative technology for active noise control [14]. Despite a more solid and lightweight design, the relatively small bandwidth of operation limits their application.

In this perspective, the development of a new type of electroacoustic transducer is of high importance to satisfy most of the requirements for various active control applications. Plasma technology appears to be a promising direction for membraneless electroacoustic transduction. This original technology has been studied in different domains related to acoustics, such as flow control [15] and sound diffusion [16]. Indeed, plasma actuators do not exhibit some drawbacks of conventional membrane-based transducers. Their construction is rather simple: it consists of two or more metallic electrodes separated by an air gap and does not involve any moving parts. As a consequence, they are less fragile, and provide a faster acoustic response to applied electrical signal over a wider frequency range of operation.

Plasma actuators can be classified in two categories according to their interaction with the surrounding gas: hot-plasma and cold-plasma actuators. The first type is typically based on dc arc discharges or high-frequency discharges [17-19]. In these devices the main mechanism of interaction with the surrounding fluid (thus interactions with the sound pressure field) is due to the heat transfer from the discharge. The most common cold-plasma actuators are Dielectric Barrier Discharge (DBD) and Corona Discharge (CD). These discharges induce so-called ionic wind that acts on the surrounding gas as a volumetric force. The additional local heat release close to the electrodes can also perturb the medium. The relative influence of electric force and 
heating on the surrounding medium is still under discussion [15,20]. Nevertheless, the heat release in cold-plasma actuators is not as preponderant as in hot-plasma and does not lead to considerable heating of closely located objects. Thus, they can be arranged on any arbitrary surface without significant impact on material integrity, which makes such actuators more preferable for active control applications. In the domain of flow control, it was shown that both CD and surface DBD are capable of generating airflows with velocities $0.1-10 \mathrm{~m} / \mathrm{s}$ over several centimeters around the electrodes [21,22]. Being attached on the surface, the actuators are able to locally reduce drag and delay the airflow laminar-turbulent transition $[15,23,24]$. The flow laminarization leads to the broadband reduction of the aerodynamic noise $[25,26]$.

All the above-mentioned works operate actuators with predefined constant parameters. To the authors' knowledge, few studies employ the transducer in a timedependent active noise control. Nasiri [27] investigated the attenuation of a single-tone acoustic noise generated by a loudspeaker in a duct using a surface DBD actuator. The aim was to cancel out the total acoustic pressure at a given position. The phase and amplitude of the actuator were adjusted to minimize the error at microphone position. Despite successful attenuation of the primary source noise, higher order harmonic components were observed in the recorded signal reducing the positive outcomes of the concept. For acoustic applications, the total harmonic distortion (THD) of the actuator should be as low as possible, otherwise it impairs the targeted noise reduction [13]. As the DBD actuator seems to be a promising candidate for active control, its THD was assessed. The experiment is reported in the Appendix A. The total harmonic distortion of the actuator exceeds $50 \%$ which makes this actuator unacceptable for active noise control application.

CD is also capable of generating sound [16, 28-30]. The distortion can stay within reasonable limits when discharge current and voltage amplitudes are limited [29]. These former results motivated us to develop a dedicated plasma-based electroacoustic transducer and analyze its acoustic properties for further implementation in active noise control systems. Positive and negative polarity CD actuators are equally often used in the literature. Nevertheless, this work is continued with the positive polarity corona discharge, since after preliminary assessments it was observed that negative polarity corona generates higher acoustic self-noise, when a constant voltage difference is applied to the electrodes (see Appendix B).

This paper investigates the sound generation process in the wire-to-mesh CD actuator. Section 2 introduces the general physical mechanism of the corona discharge. The analytical model presented in section 3 is used to derive the radiated pressure field based on the experimentally measured voltage-current characteristics of the actuator. Section 4 describes a simplified numerical model, which gives more insight on the electrical and acoustic behavior of the CD. Since it does not rely on any additional experimental data, it can be directly used for designing a corona discharge actuator as a sound generator. Finally, an experimental prototype is built and the laboratory assessments are presented in Section 5. The measurements are compared with both 
models.

\section{Corona discharge mechanism}

A CD actuator, schematically shown in figure 1, typically consists of two electrodes with at least one having a high curvature such as a needle or a thin wire. The combination of one sharp electrode (the Emitter, denoted E in figure 1) and the other with significantly larger dimensions (the Collector, denoted $\mathrm{C}$ in figure 1) improves discharge stability [15]. The air gap separating the electrodes is much larger than the characteristic radius of the emitter. When a positive DC high voltage (HV) is applied to the emitter and the collector is grounded, the electric field causes local electrical breakdown in a thin gas volume around the emitter and initiates the electron avalanches (Figure 1). In this so-called ionization layer, an accumulation of positive space charge occurs. The layer thickness is of the order of the emitter radius and depends on the applied voltage magnitude. The positive ions move within the applied external field towards the collector in the drift region. The energy gained in the electric field is not sufficient for ions to create further ionization. As a result, they transfer mechanical momentum in collisions with neutral air particles before reaching the collector. This process induces an airflow with velocities in the range $0.1-5 \mathrm{~m} / \mathrm{s}[24,31]$. The important feature of this electrohydrodynamically induced (EHD) flow is its homogeneity $[32,33]$ due to the absence of moving parts in the actuator. The airflow speed depends on the electric field strength in the drift region.

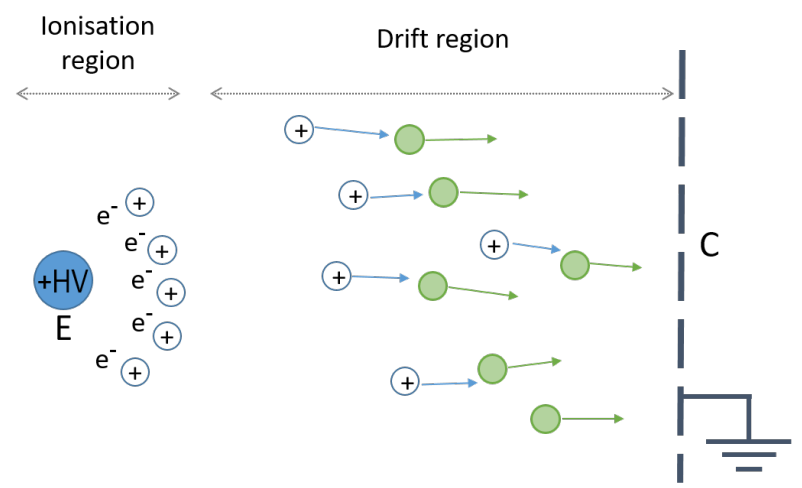

Figure 1. Mechanism of a positive corona discharge. E - emitter electrode, C collector electrode. Schematic, not to scale.

Superimposing an AC component on the constant DC voltage drop leads to a fluctuation of the electric field and, consequently, fluctuation of force acting on the ions in the drift region. Along with it, a local heat transfer from the ionization region is also subject to oscillation. These two sources cause a local pressure field perturbation and, as a result, sound waves are produced. From an acoustic viewpoint it is clear that the heat release corresponds to a monopole-type source and the fluctuating electric force to the dipolar one. These phenomena have already been modeled in the literature. 
Several analytical models were proposed which describe the sound pressure field far from the source $[16,28,29,34,35]$. The work by Matsuzawa [28] takes into account only the (dipolar) force source as the most dominant. Later studies by Bequin $[29,34]$ proved that the local gas heating also plays a non-negligible role in corona discharge sound radiation and considered both types of sources in the model. However, to set the model parameters, preliminary acoustic measurements of the frequency response and directivity are needed. In this section we propose a simple model of force and heat sources in corona discharge in wire-to-mesh geometry which requires only a measurement of voltage-current curve of the discharge to obtain all necessary parameters.

\section{Analytical model of sound generation in CD actuator}

Most of the above-mentioned works consider a point-to-grid configuration of the CD actuators. In this geometry the inter-electrode distance does not exceed $20 \mathrm{~mm}$, limiting the effective dimension of the equivalent sound source to the same value. In such a configuration, the discharge is strongly localized between the corona electrode and the nearest part of the grid [32]. Thus, the effective cross-sectional area of the discharge is limited to few square millimeters. For active control applications, a different geometry would be preferable since acoustic treatment is commonly applied with wall-mounted surface-distributed sources. Therefore, in this study we investigate a wire-to-mesh design of the CD actuator. The emitter electrodes are represented by a set of thin wires strung parallel to the collector grid as illustrated in figure 2. If the cross section of the device extends to few square centimeters with a millimeter range electrode gap, this covers a small surface area but can still be considered as a point source in the far field up to several kilohertz. Although the configuration of figure 2 inevitably leads to higher breakdown voltage and lower discharge efficiency due to the closely located emitters at the same potential, it presents several advantages. The electric field magnitude in the drift region has a more uniform distribution and can be approximated by the field in a parallel plate capacitor, simplifying the analytical description. Furthermore, the generated neutral particle velocity is expected to have the equal magnitude and be directed normally to the collector mesh over the whole cross section of the actuator in contrast to the single wire case [36]. This is convenient in an active acoustic impedance control perspective where the normal velocity of the source is controlled as a function of the input sound pressure [8].

The gas in the CD volume is weakly ionized, therefore most of the energy is transferred through elastic collisions [37]. Then, a set of linearized mass, momentum, and energy conservation equations with heat and force sources can be used to describe the particle interactions. Moreover, considering an ideal gas with adiabatic transformations, one can derive the acoustic wave equation. If the source is centered at position $\boldsymbol{r}_{\mathrm{o}}$, the solution of the wave equation in the frequency domain for an infinite 


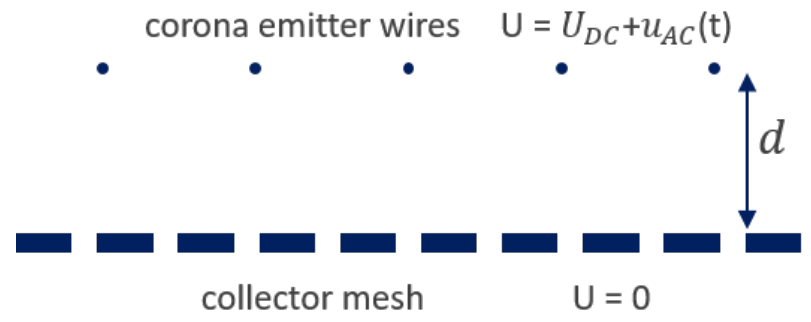

Figure 2. Sketch of a CD actuator configuration. Schematic, not to scale.

medium at position $\boldsymbol{r}$ can be expressed as [38]:

$$
p_{\omega}(\boldsymbol{r})=\iiint\left[\Phi_{\omega}\left(\boldsymbol{r}_{\mathrm{o}}\right)-\operatorname{div} \boldsymbol{f}_{\omega}\left(\boldsymbol{r}_{\mathrm{o}}\right)\right] g_{\omega}\left(\boldsymbol{r} \mid \boldsymbol{r}_{\mathrm{o}}\right) d v_{\mathrm{o}},
$$

with

$$
\Phi_{\omega}\left(\boldsymbol{r}_{\mathrm{o}}\right)=-\mathrm{i} \omega \frac{\gamma-1}{c^{2}} h_{\omega} .
$$

In (1) and (2), $g_{\omega}$ denotes the free space Green's function, $\gamma$ is the isobaric coefficient, and $c$ the speed of sound in air. The integration is taken only over the discharge volume $v_{\mathrm{o}}$ as there are no sources outside this volume. The first term $\Phi_{\omega}\left(\boldsymbol{r}_{\mathrm{o}}\right)$ represents a sound source with heat power density $h_{\omega}$. The second term accounts for the volumetric force $\boldsymbol{f}_{\omega}=\rho_{\mathrm{i}} \boldsymbol{E}$ creating the EHD flow in the drift region $\left(\rho_{\mathrm{i}}\right.$ is the positive ion space charge density and $\boldsymbol{E}$ - the electric field). The force is directed from the wires toward the collector mesh as the electric field vector. The source region can be reasonably assumed small relative to the wavelength $(k a<<1)$ up to several kilohertz. Thus, the sound pressures produced by the variation of heat power $p_{\mathrm{h}}$ and force sources $p_{\mathrm{f}}$ can be deduced accordingly:

$$
\begin{aligned}
& p_{\mathrm{h}}(r)=-\frac{\mathrm{i} \omega(\gamma-1)}{4 \pi r c^{2}}\left(\iiint h_{\omega} d v_{\mathrm{o}}\right) \exp (\mathrm{i} k r), \\
& p_{\mathrm{f}}(r)=-\frac{\mathrm{i} k}{4 \pi r}\left(\frac{\mathrm{i}}{k r}+1\right)\left(\iiint f_{\omega} d v_{\mathrm{o}}\right) \cos \theta \exp (\mathrm{i} k r) .
\end{aligned}
$$

In (3), $\theta$ is the angle between the force direction and the direction of observation. The total sound pressure can be obtained by summing the two terms: $p_{\text {tot }}=p_{\mathrm{h}}+p_{\mathrm{f}}$. Let us denote $H_{\omega}=\iiint h_{\omega} d v_{\mathrm{o}}$ and $F_{\omega}=\iiint f_{\omega} d v_{\mathrm{o}}$ representing the cumulative heat power and force over the whole discharge zone. To estimate their amplitudes, let us consider these quantities in the time domain. At a particular moment $t$, the heat power $H(t)$ can be approximated by its upper limit as the total Joule losses $H(t) \approx U(t) I(t)(U(t)$ being the applied voltage and $I(t)$ the total current in the actuator). Now we can make use of the voltage-current characteristics of the $\mathrm{CD}$ actuator, which can be written in the Townsend's form [37]: $I=C U\left(U-U_{0}\right)$, where $U_{0}$ designates the breakdown voltage of the corona discharge, and $C$ is a dimensional constant depending on gas physical parameters. It yields:

$$
H(t)=U(t) I(t)=C U(t)^{2}\left(U(t)-U_{0}\right) .
$$


The total voltage can be expressed as a sum of DC and AC components $U=$ $U_{D C}+u_{A C}(t)$. Thus, the total heat power can be decomposed into a constant and time dependent terms:

$$
\begin{aligned}
& H(t)=C\left[U_{D C}^{2}\left(U_{D C}-U_{0}\right)+\left(3 U_{D C}^{2}-2 U_{D C} U_{0}\right) u_{A C}(t)\right. \\
& \left.+\left(3 U_{D C}-U_{0}\right) u_{A C}^{2}(t)+u_{A C}^{3}(t)\right] .
\end{aligned}
$$

The first term in (5) relates to the steady heat power created by the DC offset in the actuator and can be discarded from further consideration since we are only interested in the $\mathrm{AC}$ components for acoustic characterization. The $\mathrm{AC}$ components comprise a linear term with amplitude $\left(3 U_{D C}^{2}-2 U_{D C} U_{0}\right) u_{A C}$, and higher order terms due to the quadratic current-voltage law. For instance, if $U_{D C}$ is at least 1.5 times greater than the breakdown voltage $U_{0}$, and the alternating component $u_{A C}$ does not exceed $5 \%$ of $U_{D C}$, the second-order term amplitude is 15 times weaker than the linear one which is satisfactory from the perspective of an active noise control. Thus, only the linear term in the heat power will be considered for the estimation of $p_{\mathrm{h}}$. It is written in the frequency domain as:

$$
H_{\omega}=C\left(3 U_{D C}^{2}-2 U_{D C} U_{0}\right) u_{A C}
$$

The radiated pressure due to the volumetric force $p_{\mathrm{f}}$ of $(3)$ is related to the electric force acting on the charged particles in the drift region. The current density magnitude in the drift region can be expressed as $J=\mu_{i} \rho_{i} E$, where $\mu_{\mathrm{i}}$ is the mobility of positive ions. It follows that the magnitude of the volumetric force directed from the emitter plane to the collector can be written as:

$$
F=\iiint \rho_{\mathrm{i}} E d v_{\mathrm{o}}=\iiint \frac{J}{\mu_{\mathrm{i}}} d v_{\mathrm{o}}=\frac{I d}{\mu_{\mathrm{i}}}
$$

Here $d$ is the interelectrode distance. The following derivation is similar to the one of the heat power term. Substituting the voltage-current relationship into (7) and separating the constant and time dependent terms yields:

$$
\begin{aligned}
& F=\frac{C d}{\mu_{\mathrm{i}}}\left[\left(U_{D C}-U_{0}\right) U_{D C}+\left(2 U_{D C}-U_{0}\right) u_{A C}(t)\right. \\
& \left.+u_{A C}^{2}(t)\right] .
\end{aligned}
$$

The first constant term corresponds to the steady EHD force in the discharge. The following terms are the first and second harmonics of the alternating force produced by applying the $\mathrm{AC}$ voltage component $u_{A C}(t)$. For the same setting as above $\left(U_{D C} \geq 1.5 U_{0}, u_{A C} \leq 0.05 U_{D C}\right)$ the nonlinear term in 8 is 25 times smaller in magnitude. The force generated by the linear component in the frequency domain is:

$$
F_{\omega}=\frac{C d}{\mu_{\mathrm{i}}}\left(2 U_{D C}-U_{0}\right) u_{A C}
$$


The equations (3) with source terms in the form (6) and (9) describe the low frequency sound pressure in the far field of the CD actuator in a wire-to-mesh geometry. The model only requires a simple measurement the of voltage-current characteristics to get the values of all necessary parameters such as the constant $C$ and the breakdown voltage $U_{0}$. After calibration, one can derive the frequency response and the directivity of the actuator. It is fair to mention again that the nonlinear components in this type of acoustic source are actually not negligible for high-fidelity audio performance. However, the estimation shows that they are sufficiently weak for active noise control applications. This simple model will be compared with numerical simulations of the discharge sound radiation in a finite-element software and with laboratory measurements in Section 5.

\section{Numerical simulation}

The majority of existing works on acoustic investigation of corona discharge are experimental and some of them provide theoretical/analytical description of the sound generation processes. Experimental studies report some dependencies of sound pressure field on the average electrical parameters as well as the geometry of the actuator. Analytical models derive the acoustic pressure in the far field of the source, however pressure and particle velocity in the near field are also of high interest. Particle image velocimetry can potentially be used for extracting particle velocity close to the electrodes, though this method is rather complicated to use with alternating flow. Then, a numerical simulation of the corona discharge, coupled with the acoustic field, presents an alternative solution. Particularly, the transient simulations can provide an insight on the distribution of electrical and acoustic parameters which are difficult to measure experimentally or derive analytically. For instance, space charge density, electric field and particle velocity with sound pressure can be evaluated in the near field of the actuator with high spatial and temporal resolution. The possibility to quickly change a single parameter (e.g. emitter radius) without influencing the others makes parametric studies in numerical simulation more effective and less uncertain due to the unavoidable presence of errors in fully experimental investigation. Although several works aimed to model noise produced by corona in the Trichel regime [39,40], to the authors' knowledge, there is no numerical simulation regarding the acoustic investigation of corona discharge when an alternating voltage is applied to the emitter. In this study we provide a transient simulation of positive corona discharge operation when it is fed with a voltage signal in the form $U(t)=U_{D C}+u_{A C}(t)$. The model describes the electrical and acoustic behavior of the actuator.

As previously mentioned, the corona discharge volume can be divided into two zones: ionization and drift regions. The physics of the ionization region is quite complex and taking it into account in the transient model may lead to an unacceptable computational time. Therefore, the ionization region has been discarded from the simulation, and its thickness is assumed equal to zero. In turn, the positive ions are explicitly injected from the emitter electrode which was proven to be an optimal 
alternative to the complete modelling of the plasma physics $[32,36,41,42]$.

The numerical simulation of the corona discharge is a multiphysics problem and requires considering several processes. First, the electrical potential field should be calculated. Simultaneously, the charge transport in the electric field must be taken into account. Then the induced EHD airflow is typically calculated. As mentioned above, the flow velocity does not exceed a few meters per second and cannot perturb considerably the sound generation. Thus, we suggest to model acoustic fields directly without flow, taking into account all sound sources calculated in the preceding electrical simulation step. If the distribution of static pressure and flow velocity is needed, it can be computed separately as a stationary problem.

\subsection{Governing equations and boundary conditions}

The governing equations for electrostatics and charge transport problem are the following. The distribution of electrical potential $V$ in the air is described by the Poisson equation:

$$
\nabla \cdot\left(-\epsilon_{0} \epsilon_{\mathrm{r}} \nabla V\right)=\rho_{\mathrm{i}},
$$

where $\epsilon_{0}$ is electrical permittivity in vacuum, $\epsilon_{\mathrm{r}}$ the relative electrical permittivity of the medium ( 1 for air) and $\rho_{\mathrm{i}}$ is the ion space charge density. The gradient of the electric potential defines the electric field vector as $\boldsymbol{E}=-\nabla V$. For simplicity, the model considers only positive ions with +1 charge number since these species constitute most of the space charge in a positive corona. Electric current density $\boldsymbol{J}$ in the corona discharge can be written in the drift-diffusion form:

$$
\boldsymbol{J}=\mu_{\mathrm{i}} \boldsymbol{E} \rho_{\mathrm{i}}-D_{\mathrm{i}} \nabla \rho_{\mathrm{i}}
$$

In (11), the first term represents the migration of ions in the electric field, where $\mu_{\mathrm{i}}$ is the ion mobility in air (assumed constant in the whole discharge volume). Its value

can be taken from available experimental data (e.g. [43]) with respect to actual humidity and static pressure. The second term describes the diffusion process, where $D_{\mathrm{i}}$ is the ion diffusion coefficient (in air $5 \times 10^{-5} \mathrm{~m}^{2} / \mathrm{s}$ ). This term is not taken into account in the simulation since its magnitude is negligible compared to the first one. The system of equations (10-11) is completed by the continuity equation (12) for ion current density:

$$
\nabla \cdot \boldsymbol{J}=0 .
$$

The sound wave equation with sources takes the form:

$$
\begin{aligned}
& \frac{1}{\rho c^{2}} \frac{\partial^{2} p}{\partial t^{2}}+\nabla \cdot\left(-\frac{1}{\rho}(\nabla p-\boldsymbol{f})\right)=Q_{\mathrm{m}}, \\
& Q_{\mathrm{m}}=\frac{\alpha}{\rho C_{\mathrm{p}}} \frac{\partial h}{\partial t}, \alpha=\frac{1}{c}\left(\frac{C_{\mathrm{p}}(\gamma-1)}{T}\right)^{0.5} .
\end{aligned}
$$

In (13), $p$ is the total acoustic pressure, $c$ is the sound velocity in air at atmospheric pressure, $\rho$ is the air density. The monopolar source $Q_{\mathrm{m}}$ is defined through a heat 
power $h$ under adiabatic conditions. The thermal expansion coefficient $\alpha$ depends on the isobaric heat capacity $C_{\mathrm{p}}$, isobaric coefficient $\gamma$, and ambient air temperature $T$. The heat power is defined as a power loss from Joule heating $h=\boldsymbol{J} \cdot \boldsymbol{E}$. The volumetric force $\boldsymbol{f}=\rho_{\mathrm{i}} \boldsymbol{E}$ is included in the model as a dipolar source. The significant advantage of numerical simulation is that both heat power and electric force are treated as space dependent, taking into account the distributions of electric field, current density and space charge from the solution of system (10-12).

The equations (10-12) require specification of electric potential and charge density on the electrodes to solve the electrostatics and the charge transport problems. Thus, the boundary conditions for the electric potential are the time dependent high voltage $U(t)$ on the corona wires and zero potential on the collector mesh. To build the boundary condition for space charge density at the corona electrode, the Kaptzov assumption is used [44]. The lowest voltage at which the corona discharge initiates (namely $U_{0}$ in the analytical model) corresponds to the critical electric field magnitude $E_{\mathrm{c}}$ at the wire surface. The assumption states that if the applied voltage exceeds $U_{0}$, the electric field on the electrode remains at value $E_{\mathrm{c}}$. It gives an indirect condition on ion space charge density $\rho_{\mathrm{c}}$ needed to be maintained at the emitter wire in order to achieve $E_{\mathrm{c}}$.

The value of critical field in dry air can be estimated with Peek's empirical formula $[45]$ :

$$
E_{\mathrm{c}}=3.1 \cdot 10^{6} \delta\left(1+\frac{3.08 \cdot 10^{-3}}{\sqrt{\delta R}}\right)[\mathrm{V} / \mathrm{m}],
$$

where $\delta$ is a coefficient depending on temperature and pressure ( 1 for normal conditions), and $R$ is the corona electrode radius. If the electrode is not perfectly smooth, the critical field reduces [37]. Furthermore, in recent works [46] it was shown that $E_{\mathrm{c}}$ significantly decreases with respect to air relative humidity $(\mathrm{RH})$. As all the experimental investigation in this study was conducted with $\mathrm{RH}=58 \%$ in the laboratory, we estimate the critical electric field as Peek's value reduced by $25 \%$ according to [46].

Since the applied voltage $U(t)$ is a function of time, the space charge density at the emitter wire $\rho_{c}$ should also vary in order to satisfy the Kaptzov assumption at every time step. To obtain this dependence we performed several stationary studies applying different constant voltages to the emitter electrode. The corresponding space charge values were obtained and then interpolated covering the whole range of possible values $U(t)$. Therefore, the boundary conditions for the electric potential are the time dependent high voltage $U(t)$ on the corona wires and zero potential on the collector mesh. The space charge density $\rho_{\mathrm{c}}$ at the emitter electrode is a function of the applied voltage $\rho_{\mathrm{c}}(U(t))$ and zero at the grounded collector.

For the acoustic equations, the electrodes surfaces are considered as sound hard boundaries, while the external boundary of the air domain is modeled with Perfectly Matched Layer (absorbant condition). 


\subsection{Implementation in COMSOL Multiphysics ${ }^{\circledR}$}

The geometry of the actuator in the simulation should represent the actual prototype built for experimental assessment (see Section 5). The cross section of the discharge was chosen to be $50 \times 50 \mathrm{~mm}$ and interelectrode distance of $6 \mathrm{~mm}$, the emitter wires diameter is $0.1 \mathrm{~mm}$. The electrode gap dimension was chosen in order for corona discharge to operate at voltages below $10 \mathrm{kV}$ (due to the high-voltage amplifier available for experiments). The number of wires distributed along $50 \mathrm{~mm}$ space will to be optimized in order to maximize the electrical current in the discharge and thus the power output. The parameter values used in the simulations and the experiments are summarized in table 1.

Table 1. Physical and geometrical parameters of the CD.

\begin{tabular}{llll}
\hline Parameter & Symbol & Value & Unit \\
\hline Positive ions mobility & $\mu_{\mathrm{i}}$ & $1.1 \cdot 10^{-4}$ & $\mathrm{~m}^{2} / \mathrm{Vs}$ \\
Voltage offset & $U_{D C}$ & 8.2 & $\mathrm{kV}$ \\
Critical electric field & $E_{\mathrm{c}}$ & $1.3 \cdot 10^{7}$ & $\mathrm{~V} / \mathrm{m}$ \\
Air mass density & $\rho$ & 1.23 & $\mathrm{~kg} / \mathrm{m}^{3}$ \\
Emitter radius & $R$ & $5 \cdot 10^{-5}$ & $\mathrm{~m}$ \\
Inter-electrode gap & $d$ & $6 \cdot 10^{-3}$ & $\mathrm{~m}$ \\
Wire length & $l$ & $5 \cdot 10^{-2}$ & $\mathrm{~m}$ \\
Width of collector & $w$ & $5 \cdot 10^{-2}$ & $\mathrm{~m}$ \\
\hline
\end{tabular}

The electrical part of the simulation was performed in a 2D geometry assuming the homogeneity in the direction along the emitter wires. This compromise was needed as the computational grid should be sufficiently fine to resolve strong potential gradients close to the emitter wires and keep an adequate computational time. Thus, the emitter wires are represented by an array of circles with corresponding diameter and the collector mesh is built with an array of 13 rectangular elements having a thickness of $1 \mathrm{~mm}$, length of $3 \mathrm{~mm}$ and spacing $1 \mathrm{~mm}$ (the geometry example can be found in figure 5). Such an array reflects the geometry of the actual perforated plate used in the experiments (figure 6 ). This electrode system is centered in a circular air domain with radius $0.1 \mathrm{~m}$, which is sufficiently large to eliminate all boundary effects.

The acoustic part of the model is simulated in 3D geometry inside the $0.1 \mathrm{~m}$ radius spherical domain. This domain is covered with another air domain with a thickness of $0.05 \mathrm{~m}$ representing a perfectly matched layer. It suppresses any reflection of sound waves back into the computational domain and allows the simulation of free field radiation. The emitter wires and collector mesh are represented by cylinders and parallelepipeds corresponding to a height of $50 \mathrm{~mm}$. All cross sectional dimensions correspond to the ones in 2D geometry. The computational mesh elements are larger in the acoustic study.

The numerical simulation was implemented in COMSOL Multiphysics ${ }^{\circledR}$ finiteelement based software. First, the electrical potential and charge transport problem 
is solved as a transient problem in 2D geometry. The computational domain consists of approximately 100'000 triangular elements. Afterwards, a 3D time dependent acoustic study is performed. It uses the solution of the previous study, extruding all needed electrical parameters around the electrodes (electric field, space charge, and current density) along the third dimension ( $\mathrm{z}$ axis in figures 4, 5) over the wire length of 50 $\mathrm{mm}$. This effectively creates an acoustic source representing a corona discharge inside a spherical domain. The computational mesh for the acoustic study consists of roughly 350 '000 elements.

\subsection{Geometry optimization}

Our interest in this study is to maximize the output acoustic power of the actuator in a wire-to-mesh geometry. Most of the actuator geometrical dimensions and physical parameters of the simulation are fixed and listed in the table 1 . The only flexibility left is the spatial arrangement of corona wires strung parallel to collector mesh.

Let us analyze the equations for heat and force sources according to the analytical model. Taking into account that $I_{D C}=C U_{D C}\left(U_{D C}-U_{0}\right)$, after few manipulations, (6), (9) can be rewritten in the form:

$$
\begin{aligned}
& H_{\omega}=3 I_{D C} u_{A C}+C U_{D C} U_{0} u_{A C}, \\
& F_{\omega}=\left(\frac{I_{D C}}{U_{D C}}+C U_{D C}\right) \frac{d u_{A C}}{\mu_{\mathrm{i}}}
\end{aligned}
$$

As can be seen in (15), for a given $\mathrm{DC}$ offset $U_{D C}$ and $\mathrm{AC}$ voltage $u_{A C}$, the source values are greater for higher $\mathrm{DC}$ current $I_{D C}$, breakdown voltage $U_{0}$, and coefficient $C$. When multiple wires form corona electrodes, the $U_{0}$ is higher than in the case of a single wire. Also, the current-voltage curve has a sharper rise with greater coefficient $C$ (e.g. [46]). However, if the number of wires is too high, the DC current at a given offset voltage $U_{D C}$ can decrease due to higher breakdown voltage. Therefore, this numerical simulation can be used to find the optimal number of emitter wires in order to have maximal discharge current and consequently output acoustic power.

The simulation was performed applying a constant voltage $U_{D C}=8.2 \mathrm{kV}$ to the corona electrodes. The $n$ electrodes were arranged symmetrically relative to the collector mesh. The interval between the wires was set as $\delta x=w / n$ (figure 2 shows an example of a 5 emitter wires arrangement). Only the potential and charge transport problems were computed in order to evaluate total discharge current. Figure 3 presents the results of the simulation.

When the number of wires increases from 1 to 3 , we observe more than a doubling of current magnitude. However, further increase leads to only a slight current increment, and arranging more than 6 (high voltage) electrodes over the $50 \mathrm{~mm}$ interval reduces $I_{D C}$. Thus, we concluded that for such geometrical configuration and offset voltage $U_{D C}$ $=8.2 \mathrm{kV}$, the highest discharge current is obtained with $n=5$ and 6 . Since it is easier to manufacture the experimental prototype with a smaller number of electrodes, $n=5$ was chosen for further simulations and experimental assessments. 


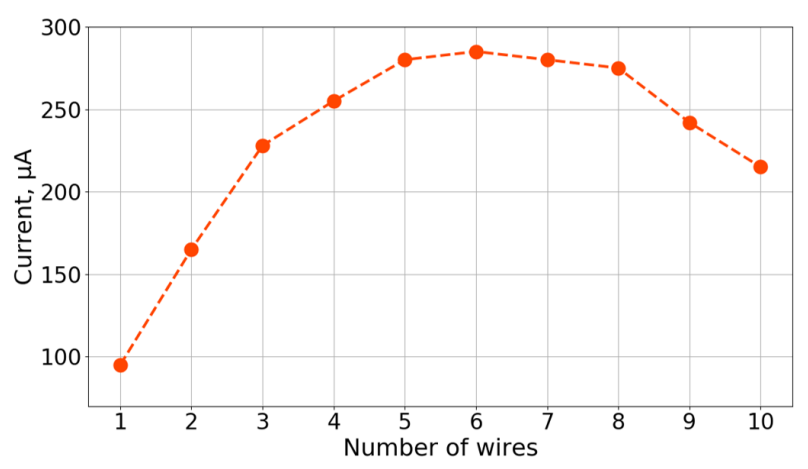

Figure 3. Total discharge current dependence on the number of emitter wires at $U_{D C}$ $=8.2 \mathrm{kV}$.

\subsection{Simulation capabilities}

The numerical model computes the radiated pressure field at an arbitrary distance from the $\mathrm{CD}$ actuator. The example of the radiation patterns at frequencies 1000 and $8000 \mathrm{~Hz}$ is presented in figure 4 . Three dimensional radiation is rather complicated to measure experimentally, so the simulation is a simple way to perform such assessment. It can be useful especially at high frequencies when the directivity strongly depends on the actuator geometry and is not symmetric around the main axis, which is the case at 8000 $\mathrm{Hz}$ in figure 4.

Besides the acoustic quantities which will be further discussed in Section 5, the simulation also gives an access to the electrical discharge parameters, such as electric potential, space charge density, electric field, and current density between the electrodes. These distributions are illustrated in figure 5 . The space charge is mostly concentrated around the emitter wires. The ions produced by each of the corona electrodes form distinct "clouds" elongated towards the grounded mesh (figure 5 - left). These clusters do not merge due to the Coulomb force acting on the particles having the same charge. The electric field is only high in the air gap between the electrodes (figure 5 - center). In contrast to the space charge density, the electric field presents a rather uniform distribution in the bulk of the discharge volume, which is clearly seen from the horizontality of potential lines. This supports the assumption made on the electric field in the analytical model. The current density (figure 5 - right) is distributed similarly to the space charge density. However, its maximal values are pulled towards the grounded mesh instead of being concentrated around corona wires as for the charge density. As the three quantities illustrated in figure 5 are linked by the relationship $J=\mu_{\mathrm{i}} \rho_{\mathrm{i}} E$ their mutual distributions become clear. Overall, these complex distributions depend strongly on the actuator geometry. For instance, if the number of emitter wires along the collector mesh is decreased, it leads to a broadening of the individual space charge clouds along the collector mesh plane. At the same time, the electric field would lose its uniformity from the left to the right. On the other hand, a higher number of corona electrodes over the same length would result in a greater uniformity of the electric field 
magnitude, but significantly increase the critical voltage of the discharge ignition (see Section 4.3).
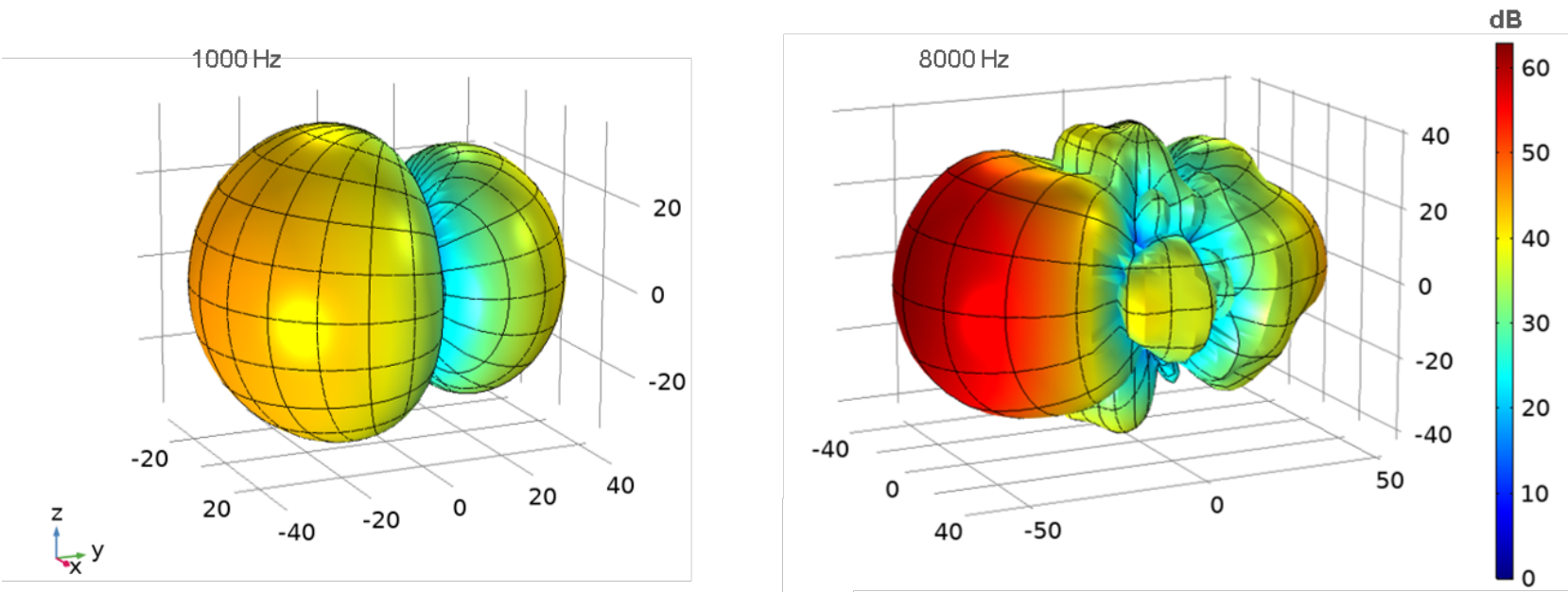

Figure 4. Three dimensional directivity patterns of the CD actuator at frequencies at $1000 \mathrm{~Hz}$ (left) and $8000 \mathrm{~Hz}$ (right). Units are indicated in dB SPL re $20 \mu P a$. The calculation is performed at the distance of $1 \mathrm{~m}$ from the actuator center. $U_{D C}=8.2$ $\mathrm{kV}, u_{A C}=300 \mathrm{Vrms}$.
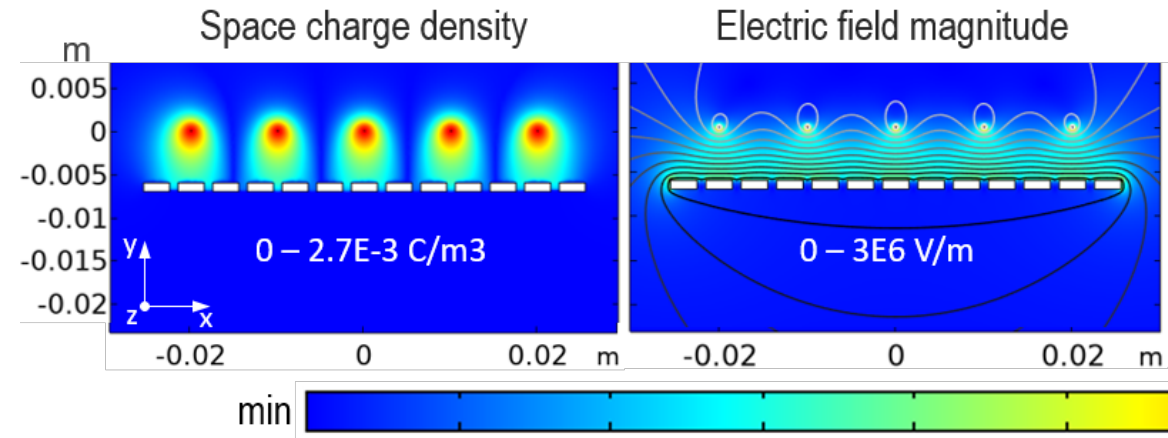

Current density magnitude

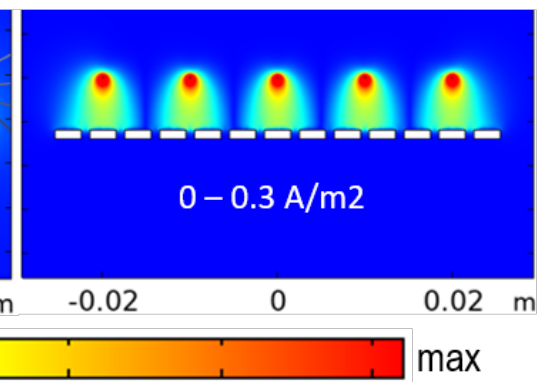

Figure 5. Space charge density, electric field magnitude with potential lines, current density magnitude distributions of the $\mathrm{CD}$ actuator. A constant voltage $U_{D C}=8.2$ $\mathrm{kV}$ is applied. The ranges indicated on pictures correspond to "min-max" colorbar, but do not represent the highest values calculated in the simulation.

\section{Experimental validation}

\subsection{Experimental setup and volt-current characteristics}

The prototype of corona discharge actuator has been built according to earlier defined dimensions and taking into account the parametric study on corona wires distribution ( Section 4.3). It consists of nichrome wire emitter ( $0.1 \mathrm{~mm}$ diameter), perforated stainless steel collector mesh, and plastic frame (figure 6). The single length of emitter wire passes 
five times across the sample hollow area. All 5 parts are parallel and separated by a distance of $10 \mathrm{~mm}$. The gap between the electrodes is $6 \mathrm{~mm}$. The total operating area is $50 \times 50 \mathrm{~mm}^{2}$. The internal edges of the plastic frame are rounded in order to inhibit a surface arc propagation between the electrodes. This configuration provides stable corona discharge in the range of $6.5-10 \mathrm{kV} \mathrm{DC}$ voltages. The actuator is powered with a TREK ${ }^{\circledR}$ 615-10 high voltage amplifier $( \pm 10 \mathrm{kV}, 10 \mathrm{~mA})$. A scheme of the acoustic measurements is presented in figure 7 . The recordings were carried out in an anechoic chamber (cut-off frequency $80 \mathrm{~Hz}$ ) using PCB 378B02 1/2" prepolarized freefield condenser microphone. The output signal to the amplifier and the acquisition with post-processing (power spectra and frequency responses) are performed by a Brüel and Kjaer Type 3160 Pulse Multichannel Analyzer.

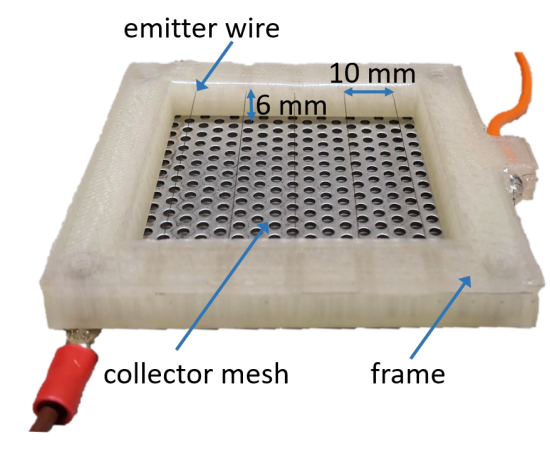

Figure 6. Photo of the corona discharge actuator.

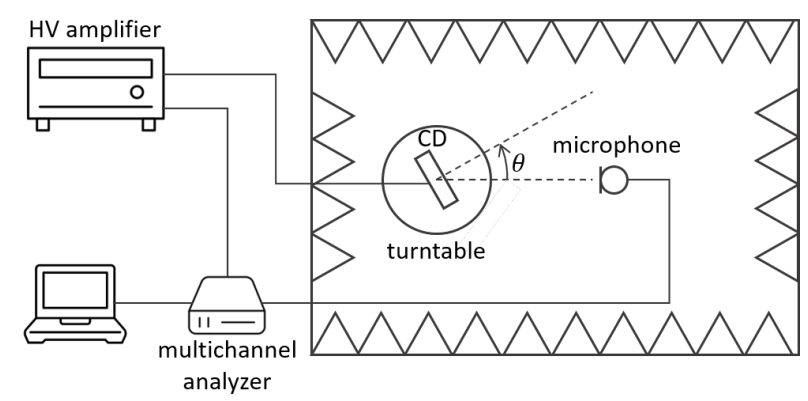

Figure 7. Scheme of the acoustic measurements setup with the CD actuator. The microphone is located at $1 \mathrm{~m}$ from the $\mathrm{CD}$ center. $\theta=0^{\circ}$ corresponds to the on-axis case. Not to scale.

Firstly, the volt-current characteristics measured experimentally were compared to the numerical model (figure 8). Both curves exhibit a prominent nonlinear behaviour. In the simulation, the current was calculated as the integral of the current density over the whole emitter surface. In the numerical simulation, the current starts flowing at lower voltages and the rise is less steep than on the experimental prototype. This mismatch could be due to several reasons. First, the 3D geometry of the actuator cannot be completely reflected with a $2 \mathrm{D}$ simulation. In addition, the ion mobility is assumed 
constant and its value is taken from the literature, considering an average $\mathrm{RH}$ for the laboratory conditions. Nevertheless, the behavior of the modelled voltage-current curve is close to the experimental one. With a DC voltage around $U_{D C}=8.2 \mathrm{kV}$ used in the acoustic experiments the current mismatch stays within $10 \%$ from 7.5 to $9 \mathrm{kV}$. Considering the above-mentioned simplifications in the numerical simulation, we can consider that it reasonably captures such phenomena as the discharge ignition and the quadratic behavior of the voltage-current characteristics.

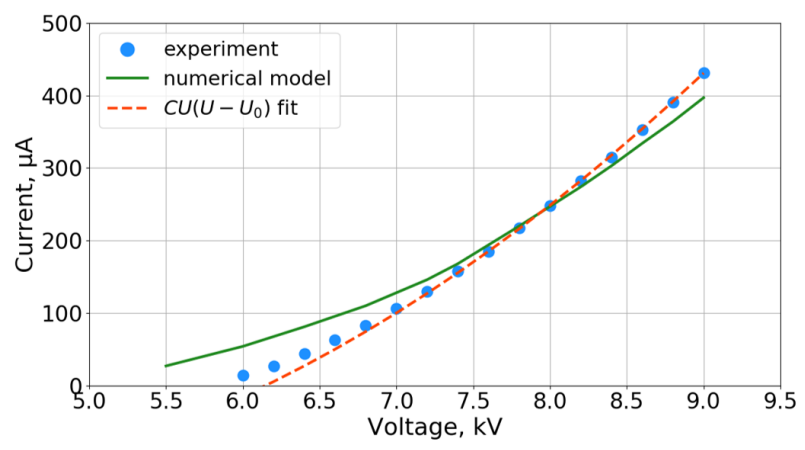

Figure 8. Voltage-current characteristics of the CD measured experimentally (blue dots), fitted with $I=C U\left(U-U_{0}\right)$ (dashed orange line), and simulated with the numerical model (green line). Fit parameters: $C=1.69 \cdot 10^{-11} \mathrm{~A} / \mathrm{V}^{2}, U_{0}=6190 \mathrm{~V}$.

\subsection{On-axis sound pressure level}

The analytical model requires the evaluation of parameters $C$ and $U_{0}$ of the volt-current characteristics in order to calculate the force and heat sources (6), (9) for the sound pressure estimation (3). These parameters were obtained by fitting the experimental curve with a Townsend's formula (dashed orange line in figure 8 ). The sound pressure levels measured experimentally and derived with both models for an AC excitation voltage of $300 \mathrm{Vrms}$ (independent of frequency), are presented in figure 9. As expected, the CD actuator does not present any resonance frequency due to the absence of moving mass. Below $4 \mathrm{kHz}$ the sound pressure levels measured experimentally increase with a $+6 \mathrm{~dB}$ /octave slope. This trend is well captured by both analytical and numerical models. It is noticeable that the curve obtained in the numerical simulation presents an approximate offset of $-1.5 \mathrm{~dB}$ compared to both the experimental and analytical results. It is likely that the mismatch observed on the numerical voltage-current curve compared to the experimental one (figure 8) is the cause of smaller current fluctuation when the discharge is driven in voltage mode. Hence, the actuator generates lower sound pressure. Above $4 \mathrm{kHz}$ the measured SPL deviates from $6 \mathrm{db} /$ octave slope. At these frequencies the actuator size can no longer be considered small relative to the sound wave length. Furthermore, the capacitance of the discharge, not taken into account in the analytical model, becomes non negligible at high frequencies [29]. Therefore, the simple analytical model fails to describe the frequency response over the high frequency range. On the other hand, the slope of the curve computed numerically decreases gradually 
at high frequencies leading to the similar dependence we observe in experiment. Since the numerical simulation is free from the above-mentioned assumptions, it describes frequency response in more detail. Finally, both models appear suitable for estimating the on-axis sound pressure level in the low frequency range.

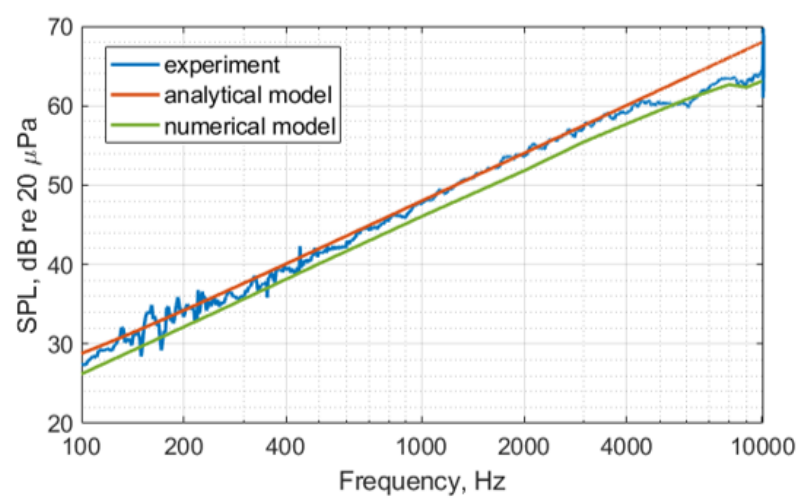

Figure 9. Sound pressure levels (SPL) of the CD actuator in the frequency range 100-10000 Hz. The microphone is located at $r=1 \mathrm{~m}$ from the actuator on the main axis, $U_{D C}=8.2 \mathrm{kV}, u_{A C}=300$ Vrms.

\subsection{Directivity}

The directivity curves at discrete frequencies (250, 500, 1000, 2000, 4000 and 8000 $\mathrm{Hz}$ ) measured experimentally and calculated with both models are shown in figure 10. Below $1000 \mathrm{~Hz}$ the patterns clearly resemble the directivity of a supercardioid, which results from the sum of the monopole and dipole. This result was also observed in other works $[29,35]$. The back radiation appears to be approximately $4 \mathrm{~dB}$ lower than on main axis. It suggests that the amplitude of the monopole source is approximately 4 times lower than that of the dipole. In addition, the relative strength of these 2 sources depends on the geometry of the actuators. As observed by Bequin et al. with a point-to-grid geometry [29], the heat source was only 2 times weaker. Thus, the local heat release in the corona discharge is non negligible and should be taken into consideration. As the frequency increases, the sound source becomes more directive. At $8 \mathrm{kHz}$ secondary lobes become clearly visible. Both analytical and numerical models are able to accurately reproduce the directivity patterns at low frequencies. Although the on-axis sound pressure levels calculated analytically match well up to $4 \mathrm{kHz}$ with the experiment, the deviations of radiation are observable already at $2 \mathrm{kHz}$ around $90^{\circ}$ and symmetrically $270^{\circ}$. Despite a slight mismatch in the overall amplitude, the numerical simulation replicates the general shape of the patterns rather well in the whole frequency range considered here. 


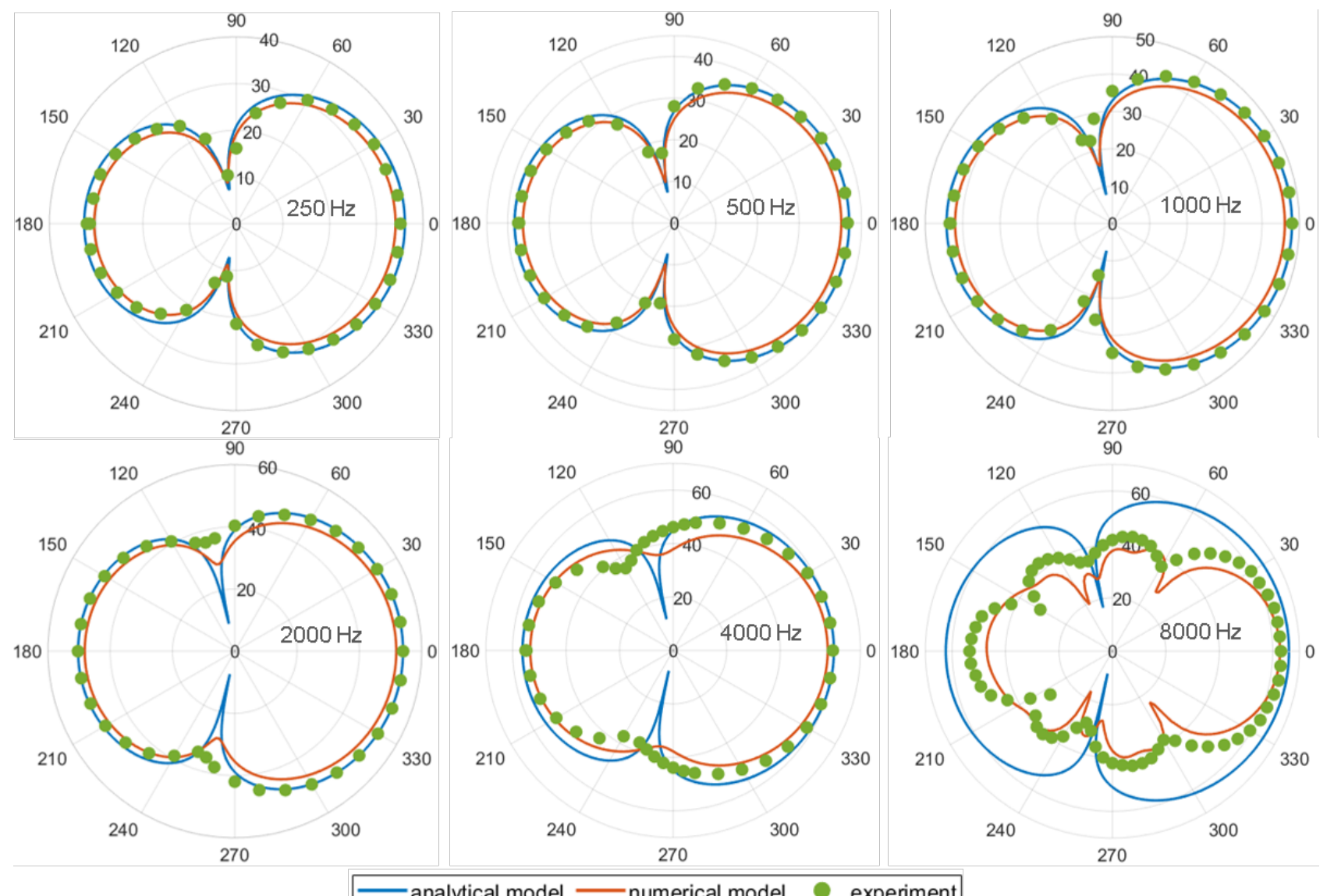

analytical model $\longrightarrow$ numerical model $\bigcirc$ experiment

Figure 10. Directivity patterns of corona discharge in the horizontal plane at frequencies $f=250,500,1000,2000,4000,8000 \mathrm{~Hz}$. Units are indicated in SPL re $20 \mu \mathrm{Pa}$ with $0^{\circ}$ corresponding to on-axis value. The microphone is located at $1 \mathrm{~m}$ from the source. Laboratory measurements are performed with $10^{\circ}$ step for $f=250$ $2000 \mathrm{~Hz}$ and $5^{\circ}$ for $f=4000,8000 \mathrm{~Hz} . U_{D C}=8.2 \mathrm{kV}, u_{A C}=300$ Vrms. Equations $(3,6,9)$ are used to calculate the patterns in the analytical model.

\subsection{Harmonic distortion}

The Total Harmonic Distortion (THD) expresses the relative contribution of higher order harmonics to the rendered acoustic pressure, when a pure sinusoidal electrical signal is applied. The THD measurements in percent measured experimentally for different excitation signal amplitudes (from 50 to $400 \mathrm{Vrms}$ ) are shown in figure 11. The distortion does not exceed $10 \%$ for the highest excitation voltage. Although this performance can be insufficient for audio applications, it can be considered as satisfactory for active noise control applications. Furthermore, to the authors' knowledge, DBD along with other types of cold plasma actuators cannot provide such low THD. Despite the local drop of THD at $f=250,4000,8000 \mathrm{~Hz}$, the distortion rate tends to grow linearly with applied voltage for most of the frequencies. 


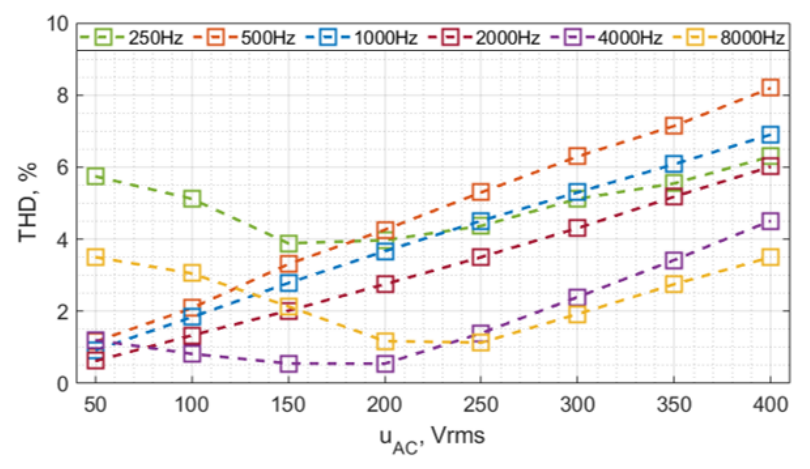

Figure 11. Total harmonic distortion of the CD actuator at frequencies $f=250,500$, 1000, 2000, 4000, $8000 \mathrm{~Hz} . U_{D C}=8.2 \mathrm{kV}$.

\section{Conclusion}

In this study we presented the experimental investigation and modelling of sound generation phenomena in a positive corona discharge. Our aim was to gain understanding of this process and to develop a dedicated actuator with geometrical configuration and relatively low distortion suitable for further implementation in active control systems.

The experimental prototype was built in a wire-to-mesh geometry with an electrode gap of $6 \mathrm{~mm}$ and cross section 50x50 $\mathrm{mm}^{2}$. The design of the experimental prototype reflects the numerical simulation results regarding the number of emitter electrodes strung parallel to the collector mesh. The idea was to increase the electrical and, consequently, the radiated acoustic power at a given $\mathrm{DC}$ voltage. On this basis, the $\mathrm{CD}$ actuator is able to generate sound pressure levels ranging from $27 \mathrm{~dB}$ at $100 \mathrm{~Hz}$ to 60 $\mathrm{dB}$ at $4000 \mathrm{~Hz}$ with $+6 \mathrm{~dB}$ /octave rise at $1 \mathrm{~m}$ distance, with an $\mathrm{AC}$ voltage $u_{A C}=300$ Vrms. With such an excitation value, the resulting harmonic distortion stays below 6 $\%$, which is satisfactory for active control applications. The THD could be potentially reduced if the actuator is driven by electrical current instead of voltage, which allows bypassing the nonlinear voltage-current relation [47].

A simple analytical model proposed in this study is able to adequately describe the acoustic field of a wire-to-mesh actuator up to $2-4 \mathrm{kHz}$. Although the theoretical models in some other studies remain valid at higher frequencies, their calibration requires directivity measurements which is not the case with the proposed model. On the contrary, in this analytical model, all unknown parameters can be retrieved with a simple measurement of voltage-current characteristics. Thus, the pressure field can be obtained without any further measurement.

The numerical simulation offers more opportunities in studying the sound pressure emission of the CD actuator. It couples the electrostatics and charge transport problem with sound radiation in a consecutive manner. Thus, the sound sources are defined on the basis of electrical solution. The simulation takes into account the geometry of the actuator and the spatial distribution of electrical parameters instead of their 
averaged values. As a result, the numerical simulation appears to be more reliable at high frequencies than the analytical model which was confirmed by comparative measurements of on-axis SPL and directivity patterns. The great value of such a numerical simulation is that one can design the actuator and evaluate sufficiently precisely its electrical and acoustic performance completely skipping the experimental phase.

The relatively low electroacoustic power efficiency of the CD actuator with the order of magnitude $10^{-5}$ was already a concern in previous works [29]. However, the total electrical power consumption is also low and equal approximately $2 \mathrm{~W}$. We presume that further studies could use the proposed numerical model to optimize the efficiency of the actuator.

If the power supply itself is not taken into account, the CD actuator presents a thickness of less than $1 \mathrm{~cm}$ and is lightweight, which can be advantageous for many applications. The electrodes can be chosen to make a visually transparent actuator if needed. Finally, it does not contain any moving parts, which could be harmed in certain harsh environments, such as aircraft engine nacelles. Therefore, the CD technology appears to be suitable for active noise control, and can offer some flexibility in design and application.

\section{Acknowledgments}

This study has received funding from the European Union's Horizon2020 research and innovation program under grant agreement No 769350.

\section{Appendix A. Harmonic distortion of DBD actuator}

A simple experiment was set up in order to assess the intrinsic harmonic distortion of the surface DBD actuator. The geometry of the flat plate DBD used in the experiment is shown in figure A1. The top HV electrode and the bottom grounded electrode are represented by the rectangles made of $17 \mu \mathrm{m}$ copper film. The dielectric separating the electrodes is made of of $1 \mathrm{~mm}$ epoxy layer. The actuator was fed with a $9 \mathrm{kV}$ peakto-peak AC voltage signal at frequency $1 \mathrm{kHz}$. In a second test, the excitation signal

was a $15 \mathrm{kHz}$ carrier frequency with the same amplitude, modulated at $1 \mathrm{kHz}$. The Sound Pressure Levels measured at $1 \mathrm{~m}$ away from the DBD actuator in an anechoic chamber (figure A2) exhibit higher order harmonics with similar amplitudes as the linear component at $1 \mathrm{kHz}$ with both excitation signals. Although the distortion in the second case appears notably lower, the THD is still higher than $50 \%$.

\section{Appendix B. Self-noise of positive and negative corona discharges}

When a constant voltage difference is applied to the actuator, the corona discharge produces a broadband acoustic noise. The total sound pressure level of such a noise 


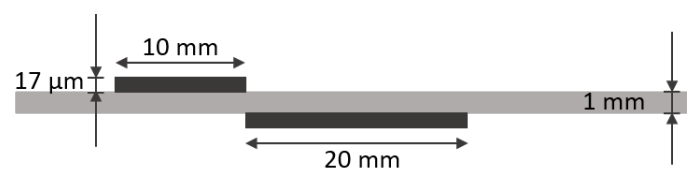

Figure A1. Schematic of the flat plate DBD actuator side view. The length of the electrodes equals to $100 \mathrm{~mm}$.

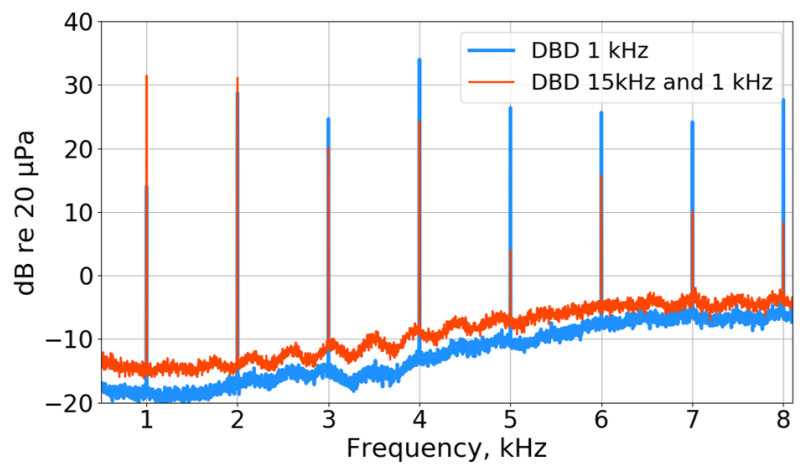

Figure A2. Sound Pressure Levels measured at $1 \mathrm{~m}$ away from a DBD actuator for different excitation signals: $9 \mathrm{kV}$ peak-peak sinusoidal voltage at $f=1 \mathrm{kHz}$ (blue line); and at $f=15 \mathrm{kHz}$ and modulated at $1 \mathrm{kHz}$ (red line).

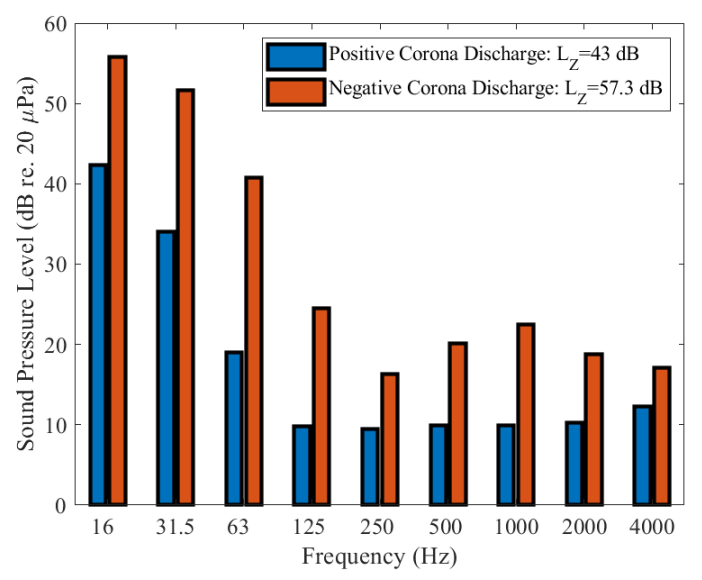

Figure B1. Self-noise levels of the positive and negative corona discharges actuators, presented by octave bands, when a constant $U_{D C}=8.2 \mathrm{kV}$ DC voltage is applied.

was assessed for positive and negative discharge polarities. The actuator used for the measurements is shown in figure 6. The experimental setup is depicted in figure 7 with $\theta$ $=0^{\circ}$. The microphone is located at $1 \mathrm{~m}$ from the source on the main axis. The constant high voltage with magnitudes $U_{D C}= \pm 8.2 \mathrm{kV}$ was applied to the corona wires in order to initiate positive and negative polarity discharges respectively.

The sound pressure levels of noise generated by the positive and negative corona discharges are presented in figure B1. Over the whole frequency range the negative corona discharge produces higher levels of noise. The total sound pressure level of 
$57 \mathrm{~dB}$ for negative corona is approximately $13 \mathrm{~dB}$ higher than in the case of positive corona and exhibits a strong audible difference. If the microphones are placed close to the actuator as in some active noise control techniques [11,12], the utilization of positive corona discharge is more advantageous due to the significantly lower noise level.

\section{References}

[1] WHO 2011 Burden of disease from environmental noise: quantification of healthy life years lost in Europe (World Health Organization. Regional Office for Europe)

[2] Allard J and Atalla N 2009 Propagation of sound in porous media: modelling sound absorbing materials (John Wiley \& Sons, 2nd ed.)

[3] Cox T and d'Antonio P 2016 Acoustic absorbers and diffusers: theory, design and application (Crc Press)

[4] Guldenschuh M, Sontacchi A, Perkmann M and Opitz M 2012 Assessment of active noise cancelling headphones 2012 IEEE Second International Conference on Consumer Electronics - Berlin (ICCE-Berlin) pp 299-303

[5] McLean I R 2000 Active noise attenuation system uS Patent 6,084,971

[6] Olson H F and May E G 1953 The Journal of the Acoustical Society of America 25 1130-1136

[7] Nelson P A and Elliott S J 1991 Active control of sound (Academic press)

[8] Rivet E, Karkar S and Lissek H 2016 IEEE Transactions on Control Systems Technology 25 63-72

[9] Fleming A J, Niederberger D, Moheimani S R and Morari M 2007 IEEE transactions on control systems technology 15 689-703

[10] Furstoss M, Thenail D and Galland M A 1997 Journal of sound and vibration 203 219-236

[11] Galland M A, Mazeaud B and Sellen N 2005 Applied acoustics 66 691-708

[12] Boulandet R, Lissek H, Karkar S, Collet M, Matten G, Ouisse M and Versaevel M 2018 Journal of Sound and Vibration 42619 - 33 ISSN 0022-460X URL http://www.sciencedirect.com/science/article/pii/S0022460X18302281

[13] Rossi M 1988 Acoustics and electroacoustics (Artech House Publishers)

[14] Sluka T, Mokrý P and Lissek H 2010 International Journal of Solids and Structures $472260 \quad-\quad 2267 \quad$ ISSN $0020-7683$ URL http://www.sciencedirect.com/science/article/pii/S0020768310001496

[15] Moreau E 2007 Journal of physics D: applied physics 40605

[16] Bastien F 1987 Journal of Physics D: Applied Physics 201547

[17] Klein S 1954 Acta Acustica united with Acustica 4 77-79

[18] Lim M 1981 Applied Acoustics 14 245-252

[19] Popov F K and Shneider M N 2017 Journal of Applied Physics 122053303 
[20] Soloviev V and Krivtsov V 2009 Journal of Physics D: Applied Physics 42125208

[21] Leger L, Moreau E and Touchard G 2001 Control of low velocity airflow along a flat plate with a dc electrical discharge Conference Record of the 2001 IEEE Industry Applications Conference. 36th IAS Annual Meeting (Cat. No. 01CH37248) vol 3 (IEEE) pp 1536-1543

[22] Berendt A, Podliński J and Mizeraczyk J 2011 Innovative dbd plasma actuators for airflow control 30th ICPIG (Citeseer) pp 28-31

[23] Peschke P 2014 Experimental investigation of pulsed dbd plasma actuators for aerodynamic flow control Tech. rep. EPFL

[24] Rickard M, Dunn-Rankin D, Weinberg F and Carleton F 2006 Journal of Electrostatics $64368-376$

[25] Thomas F O, Kozlov A and Corke T C 2008 AIAA journal 46 1921-1931

[26] Kopiev V, Kazansky P, Kopiev V, Moralev I and Zaytsev M Y 2017 Journal of Physics D: Applied Physics 50475204

[27] Nasiri A 2011 Journal of fusion energy 30 394-397

[28] Matsuzawa K 1973 The Journal of the Acoustical Society of America 54 494-498

[29] Béquin P, Castor K, Herzog P and Montembault V 2007 The Journal of the Acoustical Society of America 121 1960-1970

[30] Kopiev V, Zaitsev M Y, Kopiev V, Ostrikov N and Faranosov G 2016 Acoustical Physics 62 429-435

[31] Lacoste D, Pai D and Laux C 2004 Ion wind effects in a positive dc corona discharge in atmospheric pressure air 42nd AIAA Aerospace Sciences Meeting and Exhibit p 354

[32] Defraeye T and Martynenko A 2018 Journal of cleaner production 198 269-284

[33] Moreau E, Audier P, Orriere T and Benard N 2019 Journal of Applied Physics 125 133303

[34] Béquin P, Montembault V and Herzog P 2001 The European Physical JournalApplied Physics 15 57-67

[35] Kopiev V, Zaitsev M Y, Kopiev V, Ostrikov N and Faranosov G 2016 Acoustical Physics 62 429-435

[36] Jewell-Larsen N E, Karpov S V, Krichtafovitch I A, Jayanty V, Hsu C P and Mamishev A V 2008 Modeling of corona-induced electrohydrodynamic flow with comsol multiphysics Proceedings ESA Annual Meeting on Electrostatics, Minneapolis, Minnesota (Citeseer) pp 17-19

[37] Raizer Y P 1991 Gas discharge physics (Springer)

[38] Morse P M and Ingard K U 1986 Theoretical acoustics (Princeton university press)

[39] Zhang B, Li Z and He J 2017 Physics of Plasmas 24103521

[40] Li X, Wang J, Li Y, Zhang Q, Lu T and Cui X 2018 Physics of Plasmas 25063512 
[41] Adamiak K and Atten P 2004 Journal of electrostatics 61 85-98

[42] Ghazanchaei M, Adamiak K and Castle G P 2015 Journal of Electrostatics 73 103111

[43] Zhang B, He J and Ji Y 2017 IEEE Transactions on Dielectrics and Electrical Insulation 24 923-929

[44] Kaptsov N 1947 Moscow, OGIZ

[45] Peek F W 1920 Dielectric phenomena in high voltage engineering (McGraw-Hill Book Company, Incorporated)

[46] Nouri H, Zouzou N, Moreau E, Dascalescu L and Zebboudj Y 2012 Journal of Electrostatics 70 20-24

[47] Castor K 2001 Caractérisation des sources acoustiques associées aux décharges couronnes négatives Ph.D. thesis Le Mans 